\title{
Epirubicin plus low-dose trastuzumab in HER2 positive metastatic breast cancer
}

\author{
Alessandra Gennari $\cdot$ Michele De Tursi $\cdot$ Consiglia Carella $\cdot$ \\ Enrico Ricevuto - Cinzia Orlandini - Antonio Frassoldati · \\ Pierfranco Conte $\cdot$ Paolo Bruzzi $\cdot$ Stefano Iacobelli
}

Received: 17 March 2008/Accepted: 29 April 2008/Published online: 13 September 2008

(C) Springer Science+Business Media, LLC. 2008

\begin{abstract}
Purpose This phase II study, evaluated the activity and cardiotoxicity of first-line epirubicin plus lowdose trastuzumab (LD-T) in patients with HER2 positive MBC. Methods Patients received epirubicin $90 \mathrm{mg} / \mathrm{sqm}$ every 3 weeks plus weekly LD-T ( $2 \mathrm{mg} / \mathrm{kg}$ loading dose, then $1 \mathrm{mg} / \mathrm{kg}$ ). After $6 / 8$ cycles of epirubicin, single agent trastuzumab was continued. Cardiotoxicity was defined as signs or symptoms of congestive heart failure (CHF), or $\geq 15 \%$ decline in LVEF without symptoms, or $<15 \%$ LVEF decline to less than $50 \%$, without symptoms. Results Forty-five patients were enrolled. Twenty-three received prior adjuvant anthracyclines. Overall response rate was $61.4 \%$. The median time to progression was 7.4 months and the median survival was 32.8 months. Two (4.5\%) patients developed CHF. Conclusions Epirubicin plus
\end{abstract}

An invited commentary to this article can be found at doi:10.1007/s10549-008-0172-5.

\footnotetext{
A. Gennari $(\square) \cdot$ P. Bruzzi

National Cancer Research Institute, Largo R. Benzi, 10, 16132 Genoa, Italy

e-mail: alessandra.gennari@istge.it

M. De Tursi - C. Carella $\cdot$ S. Iacobelli

Department of Medical Oncology, University G. D’Annunzio,

Chieti-Pescara, Italy

E. Ricevuto

Department of Medical Oncology, University of L'Aquila,

L'Aquila, Italy

C. Orlandini

Division of Medical Oncology, University Hospital, Pisa, Italy

A. Frassoldati · P. Conte

Department of Medical Oncology, University of Modena

\& Reggio Emilia, Modena, Italy
}

LD-T is an active regimen, however, the relatively high rate of cardiotoxicity together with the availability of less cardiotoxic and active trastuzumab-containing combinations precludes further evaluation of this regimen.

Keywords Low-dose trastuzumab - Epirubicin · Metastatic breast cancer

\section{Introduction}

Although metastatic breast cancer (MBC) remains an incurable disease, a wide range of treatment options is available, and many further substances are currently evaluated in the clinical and preclinical setting. The decision to choose a certain therapy is usually based on tumor biology, symptoms, metastatic pattern, time to disease recurrence, and patient desire.

In human epidermal growth factor 2 (HER-2)-positive $\mathrm{MBC}$, the use of trastuzumab (T) is firmly established. Trastuzumab is a recombinant monoclonal antibody that targets the extracellular domain of HER-2 protein. Preclinical models support several potential mechanisms of action of $\mathrm{T}$, including inhibition of abnormal signaling by HER-2 overexpressing cells, activation of antibody-dependent cellular cytotoxicity, and inhibition of angiogenesis [1,2].

The addition of trastuzumab to first line chemotherapy with either paclitaxel single agent or doxorubicin plus cyclophosphamide (AC regimen) resulted into an improved activity and efficacy as compared with chemotherapy alone. Unfortunately, despite its clinical benefit, the combination of AC plus trastuzumab, was associated with an overall rate of cardiotoxicity of $27 \%$, with approximately two-third of cases belonging to New York Heart Association class III or IV [3]. 
Early clinical trials which evaluated the safety, pharmacokinetics and clinical activity of trastuzumab, used a loading dose of $4 \mathrm{mg} / \mathrm{kg}$ followed by a weekly maintenance dose of $2 \mathrm{mg} / \mathrm{kg}$ : this dose was required to obtain the minimum serum trough concentration $\left(\mathrm{C}_{\min }\right)$ of $10-20$ $\mu \mathrm{g} / \mathrm{ml}$ which was shown to be associated with preclinical activity $[1,4]$. The three-weekly schedule, based on higher doses and longer intervals, consisting in a loading dose of $8 \mathrm{mg} / \mathrm{kg}$, followed by an every-3-week maintenance dose of $6 \mathrm{mg} / \mathrm{kg}$, yielded steady state serum trough concentration roughly similar to those obtained with weekly dosing $[5,6]$. However, with both schedules, trastuzumab achieves steady state concentrations in the range of $60 \mu \mathrm{g} / \mathrm{ml}$ and a half life in excess of 3 weeks, thus exceeding the target concentration associated with clinical activity. Therefore, it can be hypothesised that the target concentration of $10-20 \mu \mathrm{g} / \mathrm{ml}$ might be achieved with lower trastuzumab doses, i.e. half of those conventionally administered. With these premises, in the attempt to improve the therapeutic index of the anthracycline/trastuzumab combination in $\mathrm{MBC}$, we performed an exploratory study to evaluate the activity and cardiac safety of epirubicin combined with low-dose trastuzumab in patients with HER-2-positive disease.

\section{Patients and methods}

\section{Study population}

Patients with histologically proven HER-2-positive MBC were enrolled into the study. HER-2 positivity was defined as either an immunohistochemical staining of $3+$ by Herceptest (Dako) or gene amplification by fluorescence in situ hybridization (FISH). In patients with tumors scored as $2+$ by Herceptest, confirmatory FISH was performed. The patients were required to have measurable disease according to WHO criteria, and an Eastern Cooperative Oncology Group (ECOG) performance status $\leq 2$. Lactating or pregnant women were excluded from the study. Patients of child-bearing potential were required to have a negative pregnancy test and to make use of effective contraception. Patients were excluded if they had received prior therapy with trastuzumab, prior chemotherapy for $\mathrm{MBC}$ or any investigational agent within 30 days before the study began. Prior adjuvant/neoadjuvant chemotherapy was allowed if terminated at least 1 year before study entry. Patients could have been treated up to a maximum of $480 \mathrm{mg} / \mathrm{sqm}$ of epirubicin and $240 \mathrm{mg} / \mathrm{sqm}$ of adriamycin. Prior endocrine therapy for adjuvant and/or for metastatic disease and prior radiation therapy were allowed. Patients had to be 18 years old, have a life expectancy longer than 6 months, and a left ventricular ejection fraction (LVEF) $>50 \%$ by echocardiography or multigated radionuclide angiography (MUGA) scan. Baseline normal haematological, renal, liver enzymes were also required.

All patients provided signed informed consent. The study was conducted in accordance with the ethical principles that originated in the Declaration of Helsinki and with local research ethics board approval at each participating centre.

\section{Treatment}

All patients received epirubicin at $90 \mathrm{mg} / \mathrm{sqm}$ as a short i.v. bolus on day 1 every 3 weeks and trastuzumab at $2 \mathrm{mg} / \mathrm{kg}$ i.v. on day 1 of week 1 and thereafter at $1 \mathrm{mg} / \mathrm{kg}$ once weekly (LD-T). After a maximum of eight cycles of epirubicin, patients received trastuzumab LD-T as a single agent for up to 52 weeks.

Treatment was discontinued for disease progression, excessive toxicity or in patients developing protocoldefined cardiotoxicity. Chemotherapy was delayed for a maximum of 2 weeks if the absolute neutrophil count was $<1.5 \times 10^{9} / 1$, if the platelet count was $<100 \times 10^{9} / 1$ or the haemoglobin was $<10 \mathrm{~g} / \mathrm{dl}$ on the day of the planned chemotherapy administration. In case of anemia, thrombocytopenia or non-hematologic grade 3 toxicity, subsequent chemotherapy was administered with a $25 \%$ dose reduction of epirubicin, within a maximum of 2 weeks. If grade 3 toxicity did not resolve within this period, patients were withdrawn from the study. In case of any non-hematological toxicity of grade 4 , epirubicin was reduced by $50 \%$ or hold at physician's judgment. Cardiotoxicity was defined as signs or symptoms of congestive heart failure $(\mathrm{CHF})$, a decline of LVEF $\geq 15 \%$ without symptomatic CHF, or a decline of LVEF less than $15 \%$ to below lower limits of normal $(50 \%)$, without symptoms. Physical examination, routine blood chemistry, chest radiography, bone scan, liver ultrasound, electrocardiogram (ECG) and echocardiogram or MUGA scan were conducted at baseline and at regular intervals throughout the study. In particular, ECG was repeated before each treatment cycle, and echocardiogram or MUGA scan was repeated every other cycle during chemotherapy and every 2 months during trastuzumab alone, or as clinically indicated. Toxicity of treatment was categorized according to NCI-CTC version 2 grading system.

Study outcome variables

The primary study objective was to evaluate overall response rate (RR) in patients treated with epirubicin plus LD-T. Imaging procedures to assess tumor response were repeated every 6 weeks. Response was evaluated via bi-dimensional measurement changes using RECIST criteria [7]. Patients who achieved a complete or partial 
response were re-imaged 4 weeks later to confirm the initial observation. Stable disease was defined as failure to achieve a response or failure to demonstrate progressive disease following two cycles of treatment. Patients were evaluable for tumor response if they received at least 2 cycles of chemotherapy. Duration of response was defined as the length of time from date of objective response to disease progression or death due to any cause. Progression free survival (PFS) was calculated from the time of initial treatment to first evidence of progression or death due to any cause. Overall survival (OS) was determined from the time of initial treatment with study drugs to death due to any cause.

\section{Statistical analysis}

The sample size was calculated according to the Simon's two-step optimal design [8], to obtain a $90 \%$ power in order to test the null hypothesis of an ORR of $50 \%$ versus the alternative hypothesis of ORR of $\geq 70 \%$ (one-sided test, $\alpha=0.05$ ). This required a total two-stage sample of 45 patients with rejection of the null hypothesis if 26 or more patients achieved an objective response.

Under this hypothesis, 21 patients were enrolled on a first step. If $<12$ responses were observed, this step had to be performed increasing the dose of trastuzumab to $2 \mathrm{mg} / \mathrm{kg}$.

Moreover, this regimen was considered feasible if the cumulative probability of developing symptomatic cardiotoxicity (CHF) was $\leq 10 \%$ at a cumulative epirubicin dose of $720 \mathrm{mg} / \mathrm{sqm}$ and $\leq 20 \%$ at a cumulative epirubicin dose of $>720 \leq 1,000 \mathrm{mg} / \mathrm{sqm}$.

The cumulative probability of developing cardiac toxicity, while taking into account the different number of patients at the various cumulative dose levels of epirubicin, was computed as follows. According to the life-table method, for each cumulative dose level, the number of patients who subsequently experienced cardiac events and had not been treated with higher cumulative doses was divided by the total number of patients treated at that level (including patients who had been treated with higher doses). Patients who received anthracyclines in the adjuvant setting were entered at the corresponding cumulative dose. Analyses were performed using SPSS/PC +11.5 statistical software (LEAD Technologies, USA).

\section{Results}

Forty-five consecutive patients (median age, 55 years; range, 34-71 years) with HER2-positive MBC were enrolled in four centres in Italy. Twenty-three $(51.1 \%)$ patients had ER and/or PR positive tumors. Twenty-four (53.3\%) patients received prior radiotherapy, and $21(46.7 \%)$ prior endocrine therapy. Twenty-three $(51.1 \%)$ patients received prior anthracyclines. The majority of patients $(82.2 \%)$ had visceral involvement. No difference in patients characteristics were observed between patients enrolled in the different centres. Table 1 lists the characteristics of the included patients.

\section{Activity}

Overall, 44 out of 45 patients were evaluable for response. One patient had HER2-negative tumor and was not eligible for the study. In the first step of the study, 12 responses among 21 patients were recorded. These results met the requirements for study continuation with trastuzumab at the dose of $1 \mathrm{mg} / \mathrm{kg}$ once weekly. Overall objective responses were recorded in 27 out of 44 patients (61.4\%); one patient $(2.3 \%)$ achieved a complete response, and 26 $(59.1 \%)$ achieved a partial response. Eleven patients (25.0\%) had stable disease following at least two cycles of epirubicin plus LD-T. Details of treatment outcome are reported in Table 2.

At a median follow-up of 11.8 months (range 1-47.4 months), 41 patients (93.2\%) have progressed, and

Table 1 Baseline patients characteristics $(N=45)$

\begin{tabular}{|c|c|c|}
\hline \multirow[t]{2}{*}{ Characteristic } & \multicolumn{2}{|c|}{ Patients } \\
\hline & No & $\%$ \\
\hline \multicolumn{3}{|c|}{ Median age, years (range) 55 (34-71) } \\
\hline \multicolumn{3}{|l|}{ Performance status } \\
\hline 0 & 26 & 57.8 \\
\hline 1 & 17 & 37.8 \\
\hline 2 & 2 & 4.4 \\
\hline \multicolumn{3}{|l|}{ Hormonal receptor status } \\
\hline Positive (ER and/or PR) & 23 & 51.1 \\
\hline Negative & 21 & 46.7 \\
\hline Unknown & 1 & 2.2 \\
\hline Prior radiotherapy & 24 & 53.3 \\
\hline Prior endocrine therapy & 21 & 46.7 \\
\hline \multicolumn{3}{|l|}{ Prior adjuvant chemotherapy } \\
\hline $\mathrm{CMF}$ & 6 & 13.3 \\
\hline Anthracyclines & 23 & 51.1 \\
\hline \multicolumn{3}{|l|}{ Metastatic sites } \\
\hline Visceral & 37 & 82.2 \\
\hline Bone \pm soft tissues & 4 & 8.9 \\
\hline Both & 4 & 8.9 \\
\hline \multicolumn{3}{|l|}{ Number of involved sites } \\
\hline 1 & 36 & 80.0 \\
\hline 2 & 7 & 15.6 \\
\hline$\geq 3$ & 2 & 4.4 \\
\hline
\end{tabular}


Table 2 Activity and efficacy $(N=44)$

\begin{tabular}{lrrr}
\hline Parameter & No. & \multicolumn{1}{c}{$\%$} & $95 \%$ CI \\
\hline Activity & & & \\
$\quad$ Overall response rate & 27 & 61.4 & \\
$\quad$ Complete response & 1 & 2.3 & \\
$\quad$ Partial response & 26 & 59.1 & \\
$\quad$ Stable disease & 11 & 25.0 & \\
Disease progression & 5 & 11.4 & \\
$\quad$ Not available & 1 & 2.3 & \\
Progression free survival & & & \\
$\quad$ Median PFS (months) & 7.3 & & \\
$\quad$ 1-year PFS & & 32.8 & \\
Overall survival & & & \\
$\quad$ Median OS (months) & 32.8 & & \\
1-year OS & & 90.6 & \\
2-year OS & & 86.8 & \\
\hline
\end{tabular}

${ }^{a}$ One patient never started the planned treatment

12 patients $(27.3 \%)$ have died. The median PFS was 7.3 months (95\% CI, 5.8-9.3 months) and the median OS was 32.8 months (95\% CI 17.1-48.5 months) (Fig. 1).

\section{Adverse events}

Forty-four out of 45 patients were evaluable for toxicity. One patient never started the planned treatment and was not considered for toxicity. Most adverse events were mild to moderate. Grade 3/4 neutropenia, thrombocytopenia and anemia occurred in $25 \%, 7 \%$ and $2 \%$ of the patients, respectively. One case of febrile neutropenia was registered. Non-hematologic toxicity was generally mild; grade 3 alopecia occurred in $46 \%$ of the patients (Table 3 ).

Patients received a median of 6 (range 1-8) threeweekly administrations of epirubicin and 32 weekly administrations of LD-T. Thirty-three $(75.0 \%)$ patients received at least six courses of epirubicin. Of the 326 courses of epirubicin administered, dose reductions or delays were necessary in 4 and 39 cycles, respectively.

\section{Cardiotoxicity}

The mean LVEF at baseline was $67.3 \%(S D=8.0 \%$; median $68.0 \%$ ). Mean LVEF was $53.5 \%(S D=12.1 \%$; median 60\%) after 6-12 months, for an absolute decline in LVEF of $20.5 \%$ from baseline $(P<0.002$ by MannWhitney) (Fig. 2).

Overall, eight episodes of protocol-defined cardiotoxicity were observed: six patients (13.6\%) showed a decline in $\mathrm{LVEF} \geq 15 \%$ as compared to baseline, while maintaining an absolute LVEF greater than $50 \%$. Two patients (4.5\%) developed symptomatic CHF after a median cumulative
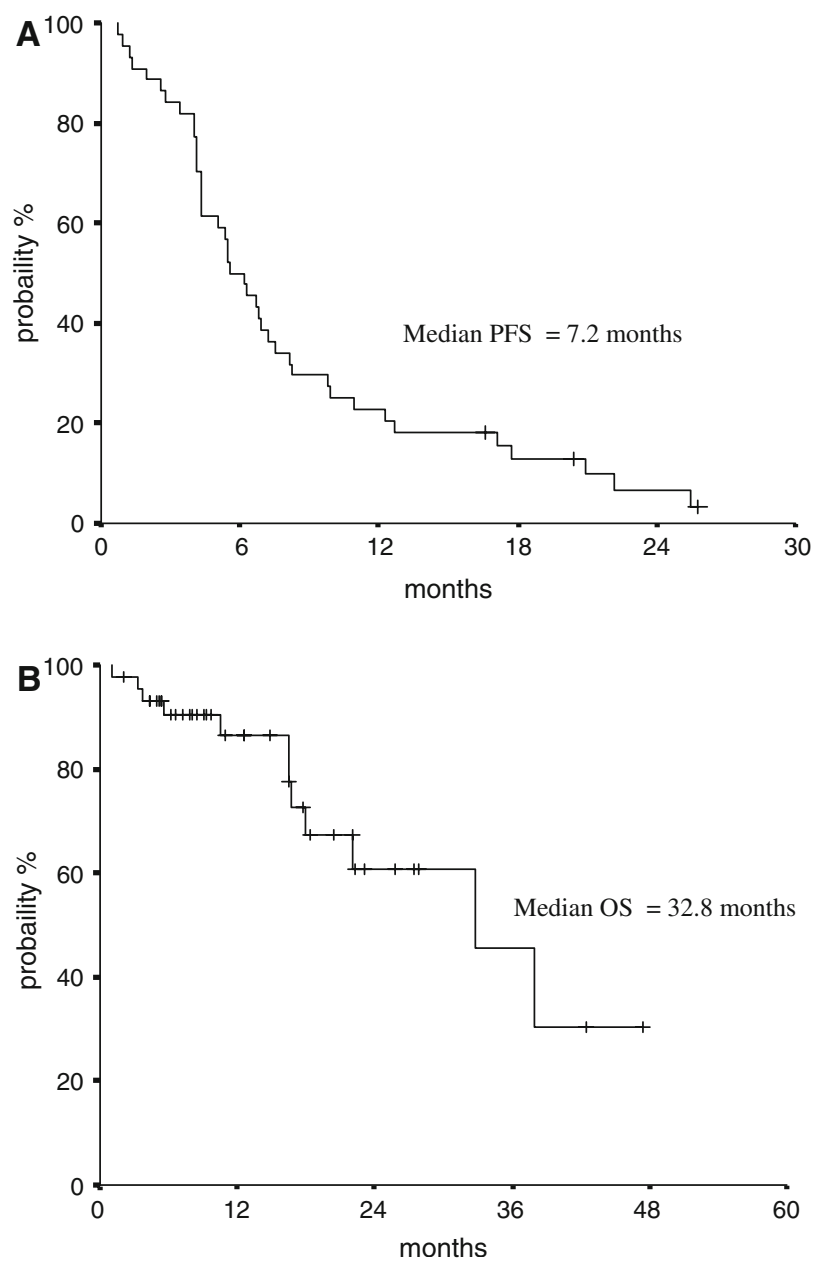

Fig. 1 (a) Progression free survival; (b) overall survival

Table 3 Incidence of hematological and not-hematological toxicities by grade $(N=44)$

\begin{tabular}{lcccc}
\hline Toxicity (\% of patients) & Grade 1 & Grade 2 & Grade 3 & Grade 4 \\
\hline Leucopenia & 18.2 & 54.5 & 6.8 & 4.5 \\
Neutropenia & 25.0 & 25.0 & 11.4 & 13.6 \\
Anemia & 27.3 & 20.5 & 2.3 & - \\
Thrombocytopenia & 4.5 & - & 6.8 & - \\
Nausea, vomiting & 11.4 & 13.6 & - & - \\
Mucositis & 6.8 & - & 4.5 & - \\
Cutaneous & - & 2.3 & 2.3 & - \\
Diarrhoea & 4.5 & 4.5 & - & - \\
Alopecia & 6.8 & 25.0 & 45.5 & - \\
\hline
\end{tabular}

epirubicin dose of $720 \mathrm{mg} / \mathrm{sqm}$ : both patients had not received anthracyclines in the adjuvant setting. The first patient was a 43-year-old woman with family history for cardiac disease and a LVEF of $60 \%$ at baseline; she developed symptomatic CHF (class III NHYA) with an LVEF of $30 \%$ after eight cycles of epirubicin plus LD-H 


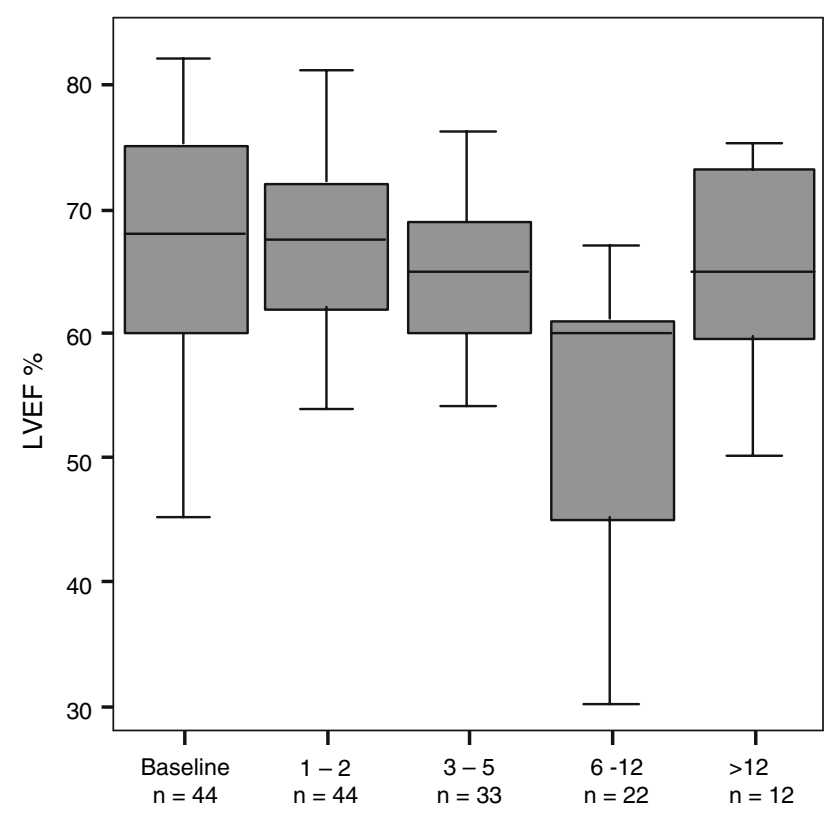

Fig. 2 Left ventricular ejection fraction modifications over time

and treatment was interrupted. She received diuretics plus an ACE-inhibitor. At most recent follow-up her LVEF improved to $40 \%$, but symptoms persisted. The second patient was a 60-year-old woman with no history of cardiac disease. Her LVEF was $69 \%$ at baseline and dropped to $56 \%$ after six cycles of epirubicin + LD-H. After cycle 8, she developed CHF (class III NHYA) with a LVEF of $38 \%$. Treatment was stopped and she was treated with diuretics plus an ACE-inhibitor. Two months later LVEF was $50 \%$ and symptoms improved. The cumulative risk of developing CHF was estimated as $8.7 \%$ at the cumulative epirubicin dose of $720 \mathrm{mg} / \mathrm{m} 2$, and the overall cumulative risk of any protocol-defined cardiotoxic event was $34 \%$ (Fig. 3).

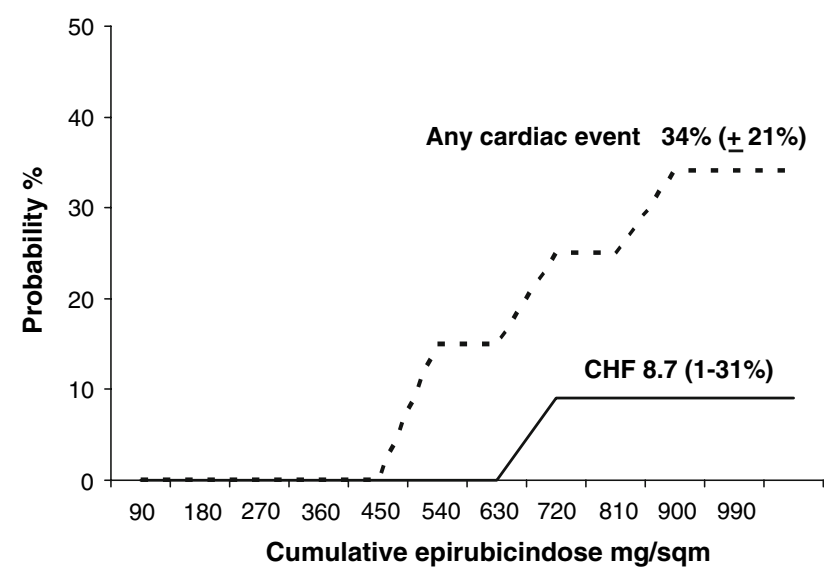

Fig. 3 Cumulative probability of CHF or $\downarrow$ L-VEF $\geq 15 \%$

\section{Discussion}

For more than 30 years, regimens containing anthracyclines have been considered among the mainstays of treatment for advanced breast cancer, with a clear therapeutic advantage over those not including anthracyclines [9]. However, the high cumulative risk of cardiotoxicity remains the major limitation to the use of these drugs [10]. The addition of trastuzumab to anthracyclines in patients with HER-2 positive breast cancer, although associated with a significantly higher clinical benefit, further increased the incidence of cardiotoxicity $[11,12]$.

In the present study, in the attempt to minimize cardiotoxicity, while maintaining antitumor activity, trastuzumab was administered at a dose $(1 \mathrm{mg} / \mathrm{kg})$ that is half of the recommended one in combination with epirubicin 90 $\mathrm{mg} / \mathrm{sqm}$. The observed response rate $(61.4 \%)$ is in the range of the activity reported with the combination of anthracyclines and trastuzumab [3]. However, two out of 44 patients (4.5\%) developed CHF, after a cumulative epirubicin dose of $720 \mathrm{mg} / \mathrm{sqm}$, for a cumulative risk of developing CHF at this dose level of $8.7 \%$. This rate is lower than the protocoldefined threshold of $10 \%$, which was chosen as maximal acceptable toxicity. Moreover, it is lower than that reported in the trial by Slamon, where the combination of anthracyclines and trastuzumab was associated with a $16 \%$ incidence of CHF. Preclinical models have shown that HER2 plays a pivotal role in limiting the left ventricular dysfunction promoted by anthracyclines. In particular, HER-2 signalling is required for the growth, repair and survival of cardiomyocytes, thus helping in maintaining cardiac contractility and function, and preventing dilated cardiomiopathy [13]. Under this hypothesis, one could speculate that the apparently decreased incidence of CHF episodes observed in the present study, can be related to an incomplete bock of the cardiac HER2 rescue pathway, occurring with reduced trastuzumab doses. Moreover, although the relationship between dose of trastuzumab and cardiac toxicity is not supported by available clinical data, in vitro data from chick embryos indicate that the drug consistently produced arrhythmia in a dose-dependent fashion [14]. Recently, updated results on the cardiac profile of the combination of epirubicin and full-dose trastuzumab as first line therapy in MBC reported a 5\% incidence of CHF, i.e. similar to our study, with an activity in the range of $60 \%$ [15]. Yet, a cardiotoxicity risk of this order of magnitude, cannot longer be considered acceptable at the light of the availability of new anthracycline formulations (e.g. liposomal doxorubicin) [16], new anti-HER-2 drugs (lapatinib) $[17,18]$ and non anthracycline based regimens (e.g. vinorelbine plus trastuzumab or taxane plus trastuzumab) [19-21], all endowed with a comparable activity, but with lower or no cardiac toxicity. 
In conclusion, the combination of epirubicin with LD-T has an activity comparable to that of anthracyclines with full-dose trastuzumab. However, this regimen is associated with a relatively high rate of cardiotoxicity, thus precluding further evaluation of this combination. Future trials in this setting should not include a control arm using conventional anthracyclines.

\section{References}

1. Vogel CL, Cobleigh MA, Tripathy D et al (2002) Efficacy and safety of trastuzumab as a single agent in first-line treatment of HER2-overexpressing metastatic breast cancer. J Clin Oncol 20:719-726

2. Hudis CA (2007) Trastuzumab-mechanism of action and use in clinical practice. N Engl J Med 357:39-51

3. Slamon DJ, Leyland-Jones B, Shak S et al (2001) Use of chemotherapy plus a monoclonal antibody against HER2 for metastatic breast cancer that overexpresses HER2. N Eng1 J Med 344:783-792

4. Cobleigh MA, Vogel CL, Tripathy D et al (1999) Multinational study of the efficacy and safety of humanized anti-HER2 monoclonal antibody in women who have HER2-overexpressing metastatic breast cancer that has progressed after chemotherapy for metastatic disease. J Clin Oncol 17:2639-2648

5. Leyland-Jones B, Gelmon K, Ayoub JP et al (2003) Pharmacokinetics, safety, and efficacy of trastuzumab administered every three weeks in combination with paclitaxel. J Clin Oncol 21:3965-3971

6. Carter P, Presta L, Gorman CM et al (1992) Humanization of an anti-p185HER2 antibody for human cancer therapy. Proc Natl Acad Sci USA 89:4285-4289

7. Therasse P, Arbuck SG, Eisenhauer EA et al (2000) New guidelines to evaluate the response to treatment in solid tumors. J Natl Cancer Inst 92:205-216

8. Simon R (1989) Optimal two-stage designs for phase II clinical trials. Control Clin Trials 10:1-10

9. Fossati R, Confalonieri C, Torri V et al (1998) Cytotoxic and hormonal treatment for metastatic breast cancer: a systematic review of published randomized trials involving 31,510 women. J Clin Oncol 6:3439-3460

10. Jones RL, Swanton C, Ewer MS (2006) Anthracycline cardiotoxicity. Expert Opin Drug Saf 5:791-809

11. Ewer MS (2007) The anthracycline-trastuzumab interaction: upregulated binding may provide vital mechanistic insight. Eur J Cancer 43:2024-2025

12. Gianni L, Salvatorelli E, Minotti G (2007) Anthracycline cardiotoxicity in breast cancer patients: synergism with trastuzumab and taxanes. Cardiovasc Toxicol 7:67-71

13. Chien KR (2006) Herceptin and the heart-a molecular modifier. N Engl J Med 354:789-799

14. Yoshiyama Y, Sugiyama T, Kanke M (2003) Cardiotoxicity of trastuzumab (herceptin) in chick embryos. Biol Pharm Bull 26:893-895

15. Untch M, Tjulandin S, Jonat W et al (2007) Cardiac safety of trastuzumab in combination with epirubicin cyclophosphamide as first-line therapy in patients with HER2 positive metastatic breast cancer. Eur J Cancer 5:216

16. Chia S, Clemons M, Martin L et al (2006) Pegylated liposomal doxorubicin and trastuzumab in HER-2 overexpressing metastatic breast cancer: a multicenter phase II study. J Clin Oncol 24:27732778

17. Burris HA, Hurwitz HI, Dees EC et al (2005) Phase I safety, pharmacokinetics, and clinical activity study of lapatinib (GW572016), a reversible dual inhibitor of epidermal growth factor receptor tyrosine kinases, in heavily pretreated patients with metastatic carcinomas. J Clin Oncol 23:5305-5313

18. Moy B, Goss PE (2007) Lapatinib-associated toxicity and practical management recommendations. Oncologist 12:756-765

19. Burstein HJ, Keshaviah A, Baron AD et al (2007) Trastuzumab plus vinorelbine or taxane chemotherapy for HER2-overexpressing metastatic breast cancer: the trastuzumab and vinorelbine or taxane study. Cancer 110:965-972

20. Esteva FJ, Valero V, Booser D et al (2002) Phase II study of weekly docetaxel and trastuzumab for patients with HER-2-overexpressing metastatic breast cancer. J Clin Oncol 20:1800-1808

21. Marty M, Cognetti F, Maraninchi D et al (2005) Randomized phase II trial of the efficacy and safety of trastuzumab combined with docetaxel in patients with human epidermal growth factor receptor 2-positive metastatic breast cancer administered as firstline treatment: the M77001 study group. J Clin Oncol 23:42654274 